\title{
El bilingüismo en la Universidad Pedagógica Nacional, Colombia: experiencias pioneras en la clase de ciencias con estudiantes de pregrado
}

\author{
BILINGUALISM IN THE NATIONAL PEDAGOGICAL UNIVERSITY, COLOMBIA: PIONEERING \\ EXPERIENCES IN SCIENCE CLASS WITH UNDERGRADUATES
}

BILINGUISMO NA UNIVERSIDADE PEDAGÓGICA NACIONAL, NA COLÔMBIA: EXPERIÊNCIAS PIONEIRAS NA AULA DE CIÊNCIAS COM ALUNOS DE GRADUAÇÃO

\author{
John Eduard Barragán Parra* / jebarragan@pedagogica.edu.co \\ Luis Alejandro Moreno Sanguino** / lasanguino0@misena.edu.co
}

\section{Resumen}

Este artículo presenta la experiencia de usar una segunda lengua para la enseñanza de la física; el ejercicio tuvo lugar en el curso de Electromagnetismo II, del Departamento de Física de la Universidad Pedagógica Nacional, y se realizó con estudiantes de IV semestre de la Licenciatura en Física. Se desarrollaron diez sesiones del curso en inglés, dedicadas al análisis del principio de inducción de Faraday desde la teoría electromagnética de campos. Dos profesores fueron los encargados de llevar a cabo las sesiones: del área de ciencias y del área de inglés. Los estudiantes estuvieron inmersos en el contexto bilingüe y se prestaron, de manera progresiva y positiva, para la comprensión del concepto de principio de inducción de Faraday, con un manejo del inglés de moderado a medio. El desequilibrio mayorante (Piaget, 1977) se presentó y permitió mayor participación y confianza a la hora de intervenir en inglés por parte de los estudiantes. La comprensión de textos originales de Maxwell (1954) se logró en una primera etapa.

\section{Summary}

The Bilingualism experience for teaching physics was carried out in the second course of electromagnetism at the physics department of the Universidad Pedagogica Nacional. It was developed with second year students of the Bachelor of Science in physics. Ten sessions of the entire course were developed in English; devoted to analyze Faradays law based in electromagnetic field point of view. One physics teacher and an English teacher were in charge of the course. The students were immersed into the bilingual context. Furthermore, they were able to understand faradays law and concepts. Understanding level was moderate-medium. The majorant disequilibrium was presented and let students take part in to the bilingual activity improving confidence between them. Originals Maxwell text has been understood in first steps.

\section{Resumo}

A experiência de usar uma segunda língua para o ensino de curso de física ocorreu em eletromagnetismo II do Departamento de Física da Universidad Pedagógica Nacional, foi realizado com alunos do quarto semestre do Curso de Licenciatura em Física. Dez sessões foram desenvolvidas em curso Inglês dedicado à análise do princípio de indução de Faraday a partir da teoria do campo eletromagnético. Dois professores estavam encarregados de realizar as sessões, a área da ciência e da área de Inglês. Os estudantes foram imersos no contexto da compreensão bilíngue, além de permitir o conceito de princípio da indução de Faraday de maneira progressiva e positiva com a gestão Inglês de moderado a médio. O desequilíbrio majorant (Piaget, 1977) está presente e permitiu uma maior participação e confiança de intervir em Inglês por estudantes. O texto original compreensão Maxwell (1954) foi obtida na primeira etapa.

Palabras clave
CLIL/EMILE, bilingüismo, elec-
tromagnetismo, enseñanza de las
ciencias.

Key words

CLIL/EMILE, Bilingualism, electromagnetism, science teaching.

Palavras chave

CLIL / EMILE, o bilinguismo, o eletromagnetismo, a educação científica.

Fecha de recepción: 10 de agosto de 2013 Fecha de aprobación: mayo 23 de 2014

\footnotetext{
* Universidad Pedagógica Nacional; Magister en Ciencias, Geofional, Universidad Nacional de Colombia.

** Servicio Nacional de Aprendizaje, SENA; Especialista en educación y docencia universitaria, Universidad la Grancolombia; estudiante de la Maestría en Lingüística y Tecnología Web, Philipps Universitat Marburg.
} 


\section{Introducción}

En el siglo XXI diferentes retos de comunicación marcan las necesidades propias de nuestros estudiantes y maestros en Colombia. Uno de estos retos está asociado a la necesidad del manejo de una segunda lengua en el campo de la enseñanza de la física en las escuelas de las poblaciones en condiciones vulnerables.

El bilingüismo forma parte fundamental de las formas de comunicación actual, en términos no solo del contacto persona a persona, sino también a través de los abundantes y distintos medios de comunicación digital. Por ejemplo, la literatura de carácter académico formal, los medios audiovisuales y la Internet, junto a las redes sociales que se desprenden de ella, son solo algunos ejemplos de dichas formas.

El origen de esta experiencia está fundado sobre dos aspectos íntimamente relacionados: primero, en el proyecto pedagógico actual de la Universidad Pedagógica Nacional, en el cual se establece que todo profesional deberá demostrar habilidades en una segunda lengua en su área de desempeño y, segundo, en el Ministerio de Educación Nacional, que, a través del Programa Nacional de Bilingüismo 2004-2019, propone para las instituciones de educación básica, media y superior.

En este estudio se realizó una propuesta pionera en el área de la educación con estudiantes universitarios, maestros en formación de la licenciatura en Física de la Universidad Pedagógica Nacional (UPN) Sede Bogotá, Colombia. Los estudiantes pertenecían al curso de Electromagnetismo II, espacio académico del ciclo de fundamentación de la Licenciatura en Física (UPN), el cual estaba orientado por el profesor John Eduard Barragán (UPN) y el profesor Alejandro Moreno (Servicio Nacional de Aprendizaje (SENA)) en las sesiones de trabajo con énfasis en el bilingüismo.

En la primera parte de este artículo presentaremos algunos estudios realizados a nivel global sobre bilingüismo y la importancia que éste desempeña en el contexto colombiano y específicamente en la UPN. En la segunda parte, se presentarán las acciones realizadas en cada sesión de nuestras prácticas (10 de 48), así como las intenciones que impulsaron la realización de dichas actividades. La tercera parte estará dedicada a la descripción de los resultados y discusión que pudimos obtener y de cómo éstos se acomodan a ciertas intenciones del trabajo, junto a la presentación de fortalezas y debilidades de la experiencia. Por último, se presentarán las conclusiones y propuestas para trabajos posteriores.

\section{Estado actual del tema}

El bilingüismo, hablar una lengua diferente a la materna o autóctona en el país de origen, es, como dice el cliché "una necesidad más que un lujo". Pero, ¿de dónde viene esa idea?; ¿por qué existe ese principio? La respuesta la podemos tomar de los estudios hechos por David Graddol en su libro English Next (2006); allí el autor hace hincapié en el desarrollo del inglés desde el siglo XIX, hasta las tendencias que se verán hacia el año 2050. Graddol (2006) explica el papel del inglés como "lingua franca1", el carácter del inglés como una habilidad básica en la educación, pero no menos importante, el papel de la misma lengua en la educación superior. Al respecto, afirma que:

"[...] el ranking mundial de universidades que provee cada año la Universidad Jiao Tong de Shanghái se ha vuelto un estándar internacional de referencia: una mirada muestra la dominación de universidades estadounidenses y universidades donde se habla inglés. De hecho, cerca de dos tercios de las 100 primeras universidades son universidades de países donde se habla inglés" (2006, p. 76).

Sumado a ello está también la gran cantidad de postgrados que se ofrecen en países no anglo-parlantes que se ofrecen en inglés. Se concluye con lo anterior que el conocimiento de una lengua extranjera es importante, no solo en términos sociales, sino en términos académicos, y no solo para acceder a la educación superior, sino para garantizar acceso a los avances científicos que la comunidad educativa ofrece.

\footnotetext{
1 Una lingua franca es una lengua que sirve como puente intercultural entre dos personas que no comparten el conocimiento de la misma lengua materna.
} 
La iniciativa del proyecto bilingüe con los estudiantes de ciencias responde a los anteriores postulados, puesto que el interés es permitir que los estudiantes de la Facultad de Ciencias de la UPN puedan tener esas habilidades en lengua extranjera, inglés en este caso, para integrarlos al contexto global y capacitarlos para responder a las características de la nueva universidad. Aunque la aproximación a la educación que se realiza en este trabajo no corresponde a las tendencias clásicas de la enseñanza de las lenguas modernas, tales como el enfoque comunicativo o el enfoque natural, en este caso se usa el método CLIL Content and Language Integrated Learning (Por sus siglas en ingles), que parte del concepto de la enseñanza de una materia de contenido, como ciencias sociales, ciencias naturales, entre otras, asociada a la enseñanza de lenguas, con el propósito de desarrollar habilidades del lenguaje a la par del contenido de la materia objeto.

CLIL fue un método desarrollado hacia la década de 1990 y se ha aplicado a nivel mundial, por ejemplo en Eslovaquia, que, después de la división de Checoslovaquia y de su ingreso a la unión europea, adoptó políticas de plurilingüismo a un mínimo de tres lenguas; para asegurarlo, se empezó en niveles escolares básicos, pero también buscando que los adultos ya tuviesen suficientes habilidades lingüísticas para apoyar dichos procesos (Pokrivcáková y Mala, 2004). Para ello, la comunidad europea pensó que CLIL sería un buen método de enseñanza, ya que es centrado en los estudiantes y se basa también en la autonomía de los mismos. Igualmente, CLIL también busca suplir otras necesidades de los estudiantes desde la perspectiva de las lenguas modernas:

Provee imput significativo y constante, ya que todas las clases se enseñan en la lengua objetivo.

Los estudiantes usan significativamente la lengua extranjera, ya sea para hacer preguntas o comentarios.

Los estudiantes usan la lengua de una manera natural, es decir, cercana a un contexto real.

Promueve la participación de los estudiantes con menos habilidades en la lengua.
Aunque se podría pensar que es demasiado laborioso para un profesor suplir las exigencias propias de un área específica, más las demandas de un profesor de lenguas, para el caso eslovaco se pensó en adecuar el currículo en las escuelas primarias, de tal manera que los profesores lograran cumplir ambos roles; sin embargo, para el caso del bachillerato, sí se requería que estuviesen capacitados en la enseñanza del Inglés para Propósitos Específicos (ESP por su sigla en inglés). Con esto en mente, el presente trabajo utiliza otro enfoque, propuesto por David Marsh (2002): mientras los estudiantes construyen sus habilidades lingüísticas en la lengua extranjera, es posible que participen en su lengua materna; esto con el fin de poder garantizar que participen activamente siempre que sea necesario.

En el caso colombiano, CLIL se ha utilizado en diferentes instituciones, pero principalmente en colegios, tales como: colegios internacionales bilingües, colegios nacionales bilingües y colegios con enfoque de inglés intensivo (Rodríguez, M., 2011). Al respecto, Rodríguez (2011) propone una serie de principios para tener en cuenta a la hora de enseñar una asignatura como ciencias, matemáticas, entre otras, usando el método CLIL:

El enfoque de la enseñanza de lenguas.

La preparación del profesor.

El desarrollo de material.

La competencia cultural e intercultural.

Aunque en su artículo la autora propone dichos parámetros en el caso de la educación básica y secundaria, hasta el momento no se encuentra información relevante sobre su aplicación en el contexto universitario colombiano. Es por esta razón que resulta pertinente el trabajo realizado en el Departamento de Física de la UPN (además de justificar la afirmación de "trabajo pionero en educación superior universitaria" mencionada más arriba), ya que si bien es importante que la educación bilingüe empiece a temprana edad, no se debe dejar de lado a los estudiantes de pregrado que aún no han desarrollado la competencia lingüística en lengua extranjera, más aún cuando serán futuros docentes. 


\section{Metodología}

El curso de Electromagnetismo II, donde tuvieron lugar las experiencias, tenía una intensidad horaria de 6 horas a la semana, los días lunes, miércoles y viernes, y en total se trabajó durante 16 semanas, es decir, 48 sesiones de clase. El trabajo teórico-práctico era trabajado dos horas a la semana, durante las sesiones de los días viernes. Todos los días viernes de las últimas 9 semanas de clase se trabajó haciendo uso de la segunda lengua, en este caso inglés, con los estudiantes del curso, es decir, 10 sesiones (aproximadamente el 20\% del total del curso). Para ello, los estudiantes estuvieron de acuerdo en realizar la actividad y en que ésta contara con un porcentaje de la nota total del curso.

En las sesiones de los viernes se trabajó sobre la concepción del principio de inducción de Faraday (Knight, 2008; Hilton, 1979; Maxwell, 1954), desde la mirada de la teoría de campos. Este concepto implicaba diferentes aspectos de tipo teórico-metodológico, como por ejemplo, la generación de campos eléctricos inducidos por variaciones del flujo magnético en espiras conductoras y en el espacio libre. Desde allí esperábamos una gran variedad de interpretaciones y prácticas con los artefactos de la vida cotidiana, por tal motivo, se escogió este concepto como el principio orientador (tópico generador) para el inicio de las experiencias con el bilingüismo en el aula de ciencias.

La primera sesión se presentó completamente en inglés, los conceptos y sus representaciones se dibujaban en el tablero de clase y se proyectaban imágenes de las representaciones de los conceptos que se trataban en dicha sesión en una pantalla de TV. Las intervenciones de los estudiantes podían ser en español como lo planteaban los programas europeos en bilingüismo Code switching, lo cual implicaba que los estudiantes podían usar su lengua materna o la lengua extranjera según fuera el caso (Marsh, 2002).

Algunos estudiantes se mostraban inquietos por la nueva experiencia en la sesión. Sin embargo, ésta transcurrió como una clase habitual, con la diferencia de que los estudiantes tenían preguntas, no solo del significado de los términos que se manejaban, sino de la temática específica de trabajo. Este punto se anota como importante, ya que prueba un cierto grado de comprensión, interés y actitud para trabajar en el aula de ciencias con una segunda lengua, incluso si no se es capaz de saber con precisión qué grado de comprensión lograron los estudiantes. Los materiales de trabajo para las actividades fueron diseñados y discutidos con anterioridad. En ellos se estableció el tipo de vocabulario a trabajar y los libros de trabajo para las primeras 7 semanas, los cuales están escritos en inglés (Knight, 2008; Maxwell, 1954).

En las sesiones posteriores (3ra, 4ta y 5ta), los estudiantes en general se lanzaron a participar en inglés. El profesor Alejandro corregía ciertos puntos sobre la pronunciación al final de las intervenciones, mientras el profesor John establecía y abría la discusión para hacer que la participación de los estudiantes no terminara y se pudiera prolongar. En algunas ocasiones, los estudiantes mezclaban el inglés y el español, es decir que podían recurrir al "Préstamo lingüístico" (Capuz, 1998), pero esto se permitía, pues de lo contrario se podría perder la confianza de los estudiantes para la participación.

Después de la sexta sesión, ya se había afianzado la confianza de los estudiantes para participar en inglés; aunque se preguntaban ciertos aspectos de forma, ya no se preocupaban en corregir aspectos de la pronunciación o el significado de las palabras, sino que se centraban en las temáticas. Esto fue posible a través de exposiciones cortas y trabajo sobre ciertas lecturas (Hewett, 1995) y pruebas realizadas en inglés de corta duración (20 o 30 minutos de las dos horas de la sesión). Así, se dio paso a una mayor intervención por parte de los estudiantes, quienes tomaron la iniciativa en la clase de los viernes, desde que entraban con el Hi teacher, hasta el I did not know how to hit that matter, entre otros. Esto, jfue emocionante en la clase de ciencias!

\section{Resultados y discusiones}

La enseñanza de la física por medio del inglés creó una necesidad comunicativa que aportó para el desarrollo de la competencia comunicativa entre los estudiantes (Hymes, 1972). Al iniciar las clases de electromagnetismo en inglés se creó un ambiente de inmersión en la 


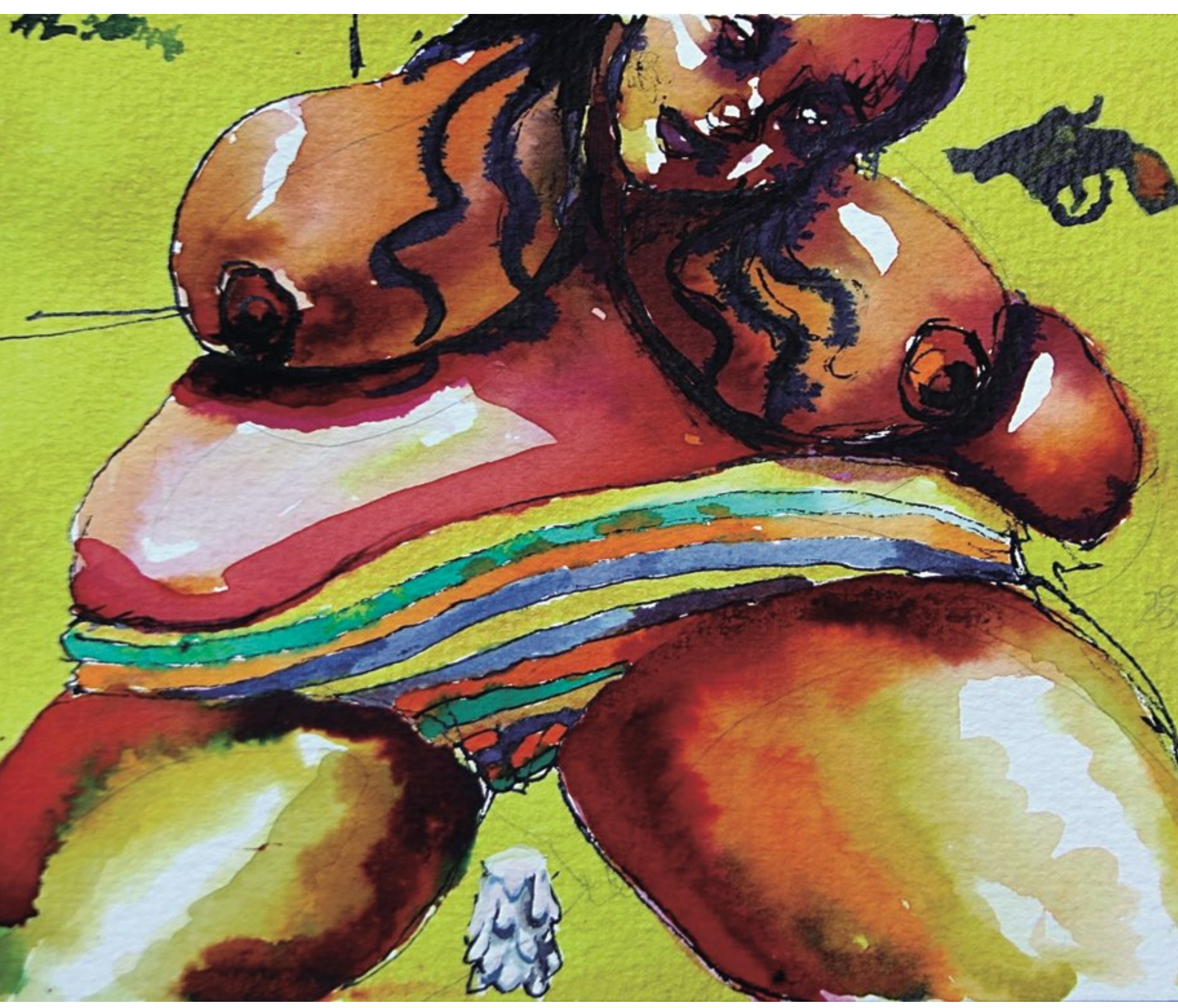

Luis Antonio Sánchez León 》 Título: La Vía Lactea ॥ Técnica: Acuarela Y Tinta » Tamaño: 17.3 X 14 Cm 》 Año: 2014 
lengua inglesa, es decir, se hizo posible abordarla desde condiciones reales o en condiciones de uso (Martínez, 2006). Sin embargo, la lengua no se enfrenta desde sus condiciones formales y estructurales, tal como se abordaría en un curso de inglés general.

Dicho ambiente generó en los estudiantes necesidades de hablar, preguntar, opinar, argumentar y ratificar sobre puntos específicos de las temáticas tratadas, en este caso, sobre el principio de inducción de Faraday. Para hacerlo, tomaban uno de dos acercamientos (Approach) dependiendo de su competencia lingüística a priori. Los estudiantes con una competencia cualitativamente alta hacían su participación directamente hacia su profesor de física (John), y quienes tenían una competencia cualitativamente baja indagaban a su profesor de inglés (Alejandro) o se apoyaban con otro compañero para luego dirigirse al profesor de física. En los dos casos, los estudiantes recibieron una retroalimentación, ya fuera antes de su participación (estudiante con baja competencia), o después de su participación (estudiantes con alta competencia). Dicha retroalimentación co-ayudó a que desarrollaran el uso de la lengua y elevaran su competencia. Por lo tanto, resultó significativa para los estudiantes.

Las discusiones con los estudiantes permitieron avanzar en la comprensión del concepto de inducción electromagnética. Diferentes prácticas de laboratorio, análisis y discusiones sobre las ideas de variación del flujo magnético y no solo de la existencia de flujo magnetico, formaron parte de dichas comprensiones. Sin embargo, no pudo alcanzarse la profundidad ideal en el análisis de los textos originales de Maxwell (1954). Solo las ideas de tubos de fuerza lograron asentarse en una primera etapa, a través de ejercicios en los cuales se pensaba en el flujo del campo usando representaciones vectoriales en las cuales NO importaba la magnitud del campo, solo el flujo y su cambio.

Las afirmaciones creaban desequilibrio mayorante (Piaget, 1977) y permitían el cuestionamiento y la intervención de los estudiantes. Este tipo de desequilibrio se presentó en varias sesiones y facilitó que los estudiantes se apropiaran de sus trabajos e hicieran sus presenta- ciones finales en inglés (Debido a la apropiación del tema y cierta libertad para poder expresar las ideas). Las presentaciones finales, en las cuales se presentaron exposiciones grupales, mostraron lo positivo de las experiencias aquí narradas. Algunas grabaciones de video podrían confirmar estas apreciaciones.

Por otra parte, la presente experiencia podría explicar la relevancia del método CLIL/EMILE frente a métodos contemporáneos, o incluso a modelos clásicos de la enseñanza de lenguas tales como el enfoque comunicativo, en este caso en la educación superior. Si bien, el enfoque comunicativo busca presentar la lengua en condiciones de uso controladas por el profesor, el CLIL presenta una situación no modelada ni controlada, sino asumida desde el estadio mismo de la necesidad lingüística del estudiante.

En otras palabras, mientras en el enfoque comunicativo el profesor presenta de una manera controlada una situación emulada y artificial (Richards, 2005), en términos de vocabulario, uso del lenguaje, funciones comunicativas, y gramaticales; en el CLIL no existe tal situación, por lo que los estudiantes buscan herramientas por sí mismos, con sus compañeros, o con el profesor de la lengua extranjera, para comunicar de una manera más efectiva y significativa sus ideas sin estar supeditados a una nota, a una corrección, o a algún elemento similar, ya que éste no es el objetivo principal de la clase.

Lo anterior se observó por medio de las interacciones y participaciones de los estudiantes, quienes al principio buscaban al profesor de inglés para apoyarse a la hora de generar su acto de habla. Pero luego, los mismos estudiantes buscaban recursos propios, e incluso los mismos compañeros con una competencia lingüística superior servían de puente y/o apoyo, generando así prácticas de aprendizaje cooperativo (Slavin, 1995; Baquero, 1996). A su vez, tanto los estudiantes con una competencia comunicativa alta, como aquellos con bajos índices de competencia, afirmaron beneficiarse de la práctica pedagógica, ya que les permitió "practicar y aprender inglés y física al mismo tiempo", y fueron ellos quienes manifestaron el avance en el campo de la segunda lengua; no obstante, algunos encontraron 
mayor dificultad para comprender los temas de la clase de contenido (electromagnetismo en este caso). Lo anterior se evidenció por medio de una encuesta aplicada a los estudiantes.

\section{Conclusiones}

La enseñanza de la física a través del uso del inglés, permitió desarrollar habilidades comunicativas en segunda lengua entre los estudiantes del curso de electromagnetismo II, de la carrera de Licenciatura en Física de la Universidad Pedagógica Nacional. El concepto que en particular se abordó fue el principio de inducción de Faraday desde la teoría electromagnética de campos.

La inmersión en una segunda lengua para la enseñanza de la física, desarrollada en este estudio, se diferencia del método tradicional de aprendizaje de la física y del inglés a través de las formas estructuralista y secuencial (Richard, 2005). Esta metodología (CLIL) permitió que los estudiantes se interesaran en la participación y, para este estudio, mostraran actitudes positivas frente a la clase de ciencias.

Las discusiones con los estudiantes permitieron la comprensión y apropiación del concepto de inducción electromagnética desde la teoría de campos, esto lo evidenció la forma en que hablaban del fenómeno y las relaciones que establecían con sus compañeros en sus intervenciones orales. Además, hicieron posible mejorar la comprensión y habilidades lectoras de libros de texto escritos en inglés en el área de ciencias (Knight, 2008). Sin embargo, la lectura de originales (Maxwell, 1954) solo se logró en una primera etapa, debido a que los tiempos de trabajo fueron reducidos.

La metodología CLIL/EMILE (Marsh, 2002), mostró funcionar satisfactoriamente en este estudio. Trabajos posteriores podrían afianzar o rebatir dicha afirmación. Es posible implementar el uso de una segunda lengua (bilingüismo) en los cursos de los programas curriculares de la Universidad Pedagógica Nacional. Este estudio se realizó con el $20 \%$ del total del período académico dedicado al curso de Electromagnetismo II.

\section{Agradecimientos}

Agradecemos a la Universidad Pedagógica Nacional por permitirnos pensar en estos temas y, especialmente, al señor Rector de la UPN, Profesor Juan Carlos Orozco Cruz, por su idea propositiva inicial para la puesta en marcha de este trabajo.

\section{Referencias}

Baquero, R. (1996). Vigostky y el aprendizaje escolar. Argentina: Editorial Aique, p. 137.

Capuz, J. G. (1998). El Préstamo Lingüístico: conceptos, problemas y métodos. Valencia: Universidad de Valencia.

Graddol, D. (2006). English Next. Lóndes: British Council, p. 76.

Holton, G. (1979). Introducción a los conceptos y teorías físicas de las ciencias físicas. Barcelona: Editorial Reverté.

Hewitt, P. G. (1995). Física Conceptual. USA: Addison-Wesley Iberoamericana.

Knight, R. D. (2008). Physics: for scientists and engineers; second edition/A strategic Approach. USA: Addison-Wesley.

Maxwell, J. C. (1954). A treatrice on electricity and magnetism. New York: Dover Publications Inc.

Martínez, L .M. (2006). Comunicación y lenguaje: competencia comunicativa, supuestos de los profesores de Básica Primaria. Bogotá: Centro Editorial Universidad del Rosario, p. 56.

Marsh, D. (2002). CLIL/EMILE. The European Dimension. Finland: Unicom, Continuing Education Centre, p. 17.

Piaget, J. (1977). The role of action in development of thinking. En Overtone, W. F., y Gallaher, J. M. (Eds.). Knowledge development: Vol. I. Advances in research and theory. H. Furth, trans., 17-42.

Pokrivcáková, S., y Mala, E. (2004). Developing Plurilinguism in Slovakia: The CLIL Method. A Starting Point. Revista Profile. Vol. 5, No. 1., p.117. Bogotá: Universidad Nacional de Colombia.

Richards, J. (2005). New interchange intro. Cambridge: Cambridge University Press, 10- 20.

Rodríguez, M. (2011). CLILL: Colombia Leading Into Content Language Learning. Ikala Revista de Lenguaje y Cultura. Vol. 16, No 28, p. 80. Medellín: Universidad de Antioquia.

Slavin, R. (1995). Aprendizaje Cooperativo. Teoría, investigación y práctica. Buenos Aires: Editorial Aique, p. 49. 


\section{Diálogo del conocimiento}

Los profesores John Eduard Barragán y Luis Alejandro Moreno en este artículo nos dan luces sobre un elemento muy importante para el aprendizaje de saberes, como lo es la interdisciplinariedad. Se observa a lo largo de la experiencia una cooperación disciplinar que la hace interesante e innovadora, frente a prácticas universitarias de educación tradicional. Inquieta que su trabajo sea pionero en Colombia, puesto que experiencias así deberían darse permanentemente en el aprendizaje de un segundo idioma.

Esta estrategia lleva a pensar sobre la forma en la que en ocasiones los docentes olvidan la importancia de ubicar los conocimientos en un contexto significativo para sus estudiantes. Tanto en el colegio, como en la universidad se aprenden saberes que se olvidan fácilmente porque no se vuelven útiles para la vida. Es decir, son conocimientos desligados de la realidad, fragmentados, los cuales no motivan a seguir aprendiendo, sino que llevan al aburrimiento y desinterés.

En el caso del aprendizaje de una segunda lengua se hace importante reflexionar sobre la manera en la que los niños adquieren su lengua materna. Ellos están sumergidos en un contexto lleno de problemas interesantes que les crea la necesidad de hablar y memorizar cada palabra. Es así como se asegura el éxito en el aprendizaje del lenguaje en los niños, mediante dicha cooperación de disciplinas, la cual más que interdisciplinar es transdisciplinar. Los niños interiorizan a la par del lenguaje todas las materias de las que consta la vida; así, colores, sabores, personajes, lugares, e incluso movimientos se aprenden simultáneamente con el idioma.

En contraste, la forma en la que se enseña una segunda lengua, puede convertirse en una verdadera tortura para quien la aprende, puesto que hay gran información por memorizar: reglas gramaticales, vocabulario, frases idiomáticas, en fin, un mundo de conocimientos. Estos se van a olvidar fácilmente si con prontitud, no se aplican a situaciones reales.

Sin embargo, la experiencia de los profesores de la Universidad Pedagógica Nacional muestra cómo la física viene a ser aquella forma en la que los estudiantes practican una segunda lengua en un contexto significativo, en el que encuentran obstáculos y problemas que deben resolver para poderse comunicar, es así como el concepto piagetiano de "desequilibrio mayorante" tiene lugar, llevándolos cada vez a un proceso cognitivo con estados de equilibrio más complejos, es decir aprendizajes arraigados.

La estrategia de estos profesores es un buen ejemplo de cómo el aprendizaje de una segunda lengua ha de transversalizarse en los currículos, con el fin de que puedan verse mejores resultados. Claro, con el riesgo de una menor comprensión en cada una de las áreas que integren el programa, pero con el mejoramiento de la misma, a medida que se familiarizan con el nuevo idioma.

Martha Patricia Torres Sabogal 